\title{
Intraoperative neurophysiological monitoring during resection of infratentorial lesions: the surgeon's view
}

\author{
Philipp J. Slotty, MD, ${ }^{1}$ Amr Abdulazim, MD, ${ }^{1}$ Kunihiko Kodama, MD, ${ }^{2}$ Mani Javadi, MD, ${ }^{3}$ \\ Daniel Hänggi, MD, ${ }^{1}$ Volker Seifert, MD, PhD, ${ }^{3}$ and Andrea Szelényi, MD ${ }^{1,3}$
}

${ }^{1}$ Neurochirurgische Klinik, Universitätsklinikum Düsseldorf; ${ }^{2}$ Klinik für Neurochirurgie, Universitätsklinikum Frankfurt am Main, Germany; and 'Department of Neurosurgery, Shinshu University School of Medicine, Matsumoto, Japan

\begin{abstract}
OBJECTIVE Methods of choice for neurophysiological intraoperative monitoring (IOM) within the infratentorial compartment mostly include early brainstem auditory evoked potentials, free-running electromyography, and direct cranial nerve (CN) stimulation. Long-tract monitoring with somatosensory evoked potentials (SEPs) and motor evoked potentials (MEPs) is rarely used. This study investigated the incidence of IOM alterations during posterior fossa surgery stratified for lesion location.
\end{abstract}

METHODS Standardized CN and SEP/MEP IOM was performed in 305 patients being treated for various posterior fossa pathologies. The IOM data were correlated with lesion locations and histopathological types as well as other possible confounding factors.

RESULTS Alterations in IOM were observed in 158 of 305 cases (51.8\%) (CN IOM alterations in 130 of 305 [42.6\%], SEP/MEP IOM alterations in 43 of 305 [14.0\%]). In 15 cases (4.9\%), simultaneous changes in long tracts and CNs were observed. The IOM alterations were followed by neurological sequelae in 98 of 305 cases (32.1\%); $62 \%$ of IOM alterations resulted in neurological deficits. Sensitivity and specificity for detection of CN deficits were $98 \%$ and $77 \%$, respectively, and $95 \%$ and $85 \%$, respectively, for long-tract deficits. Regarding location, brainstem and petroclival lesions were closely associated with concurrent CN IOM and SEP/MEP alterations.

CONCLUSIONS The incidence of IOM alterations during surgery in the posterior fossa varied widely between different lesion locations and histopathological types. This analysis provides crucial information on the necessity of IOM in different surgical settings. Because MEPISEP and CN IOM alterations were commonly observed during posterior fossa surgery, the authors recommend the simultaneous use of both modalities based on lesion location.

http://thejns.org/doi/abs/10.3171/2015.11.JNS15991

KEY WORDS somatosensory evoked potential; motor evoked potential; infratentorial neoplasms; intraoperative neuromonitoring; brainstem cavernoma; diagnostic and operative techniques

$\mathrm{S}$ UPPORTED by profound experimental and clinical data, intraoperative monitoring (IOM) has become the standard of care during resection of intraparenchymal and critically located extraaxial brain lesions. Most publications on long-tract monitoring with somatosensory evoked potentials (SEPs) and motor evoked potentials (MEPs) focus on the supratentorial compartment, where the bulk of critical pathologies, aneurysms, and gliomas are located. 4, 10,21,22,32 There is long-standing experience with neuromonitoring of somatosensory and motor pathways regarding spine and intradural spinal pathology. ${ }^{2,12,18,24,27}$ Publications on the use of IOM in the infratentorial compartment or posterior fossa are commonly focused on cranial nerve $(\mathrm{CN})$ monitoring, because these structures are systematically at risk during manipulation even if not involved in the pathology itself., 3,25,26 Investigations of the usefulness and therapeutic yield of MEPs and SEPs during surgery in the posterior fossa are rare. Our group recently analyzed the correlation of postoperative outcome and IOM changes and found a high sensitiv-

ABBREVIATIONS BAEP = brainstem auditory evoked potential; $\mathrm{CN}=$ cranial nerve; $\mathrm{CPA}=$ cerebellopontine angle; $\mathrm{DNS}=$ direct nerve stimulation; EMG = electromyography; $I O M=$ intraoperative monitoring; $M E P=$ motor evoked potential; NPV = negative predictive value; $P P V=$ positive predictive value; SEP = somatosensory evoked potential.

SUBMITTED April 30, 2015. ACCEPTED November 11, 2015.

INCLUDE WHEN CITING Published online February 26, 2016; DOI: 10.3171/2015.11.JNS15991. 
ity and a high negative predictive value (NPV) of longtract monitoring and postoperative neurological status, ${ }^{8}$ confirming data by Neuloh et al. and Sarnthein et al..$^{20,28}$

In relating intraoperative alteration of MEPs to motor outcome, the results of all the aforementioned studies emphasized that the warning criteria for MEPs should also follow the rules that have recently been suggested for supratentorial surgeries. ${ }^{17,21,31}$ There is more and more evidence that this rule also applies for corticobulbar MEPs ${ }^{1}$ (and I. Fernández-Conejero, personal communication, 2014). A growing body of evidence suggests that even in infratentorial procedures, MEP monitoring does not follow the "all or nothing response principle" applied in spinal surgery monitoring. ${ }^{11,18}$ The question remains, in which posterior fossa surgeries are long-tract monitoring alterations common, and should MEP/SEP monitoring therefore be recommended in addition to $\mathrm{CN}$ monitoring?

In this second and more extensive analysis including data from 2 institutions, we focus on the correlation of lesion location and histopathological type with IOM alterations and their diagnostic yield. The aim is to allow an estimation of the probability of IOM alterations in different lesion locations. This knowledge can then be used to tailor IOM setups to surgical procedures in the posterior fossa and to define standardized IOM approaches based on the lesion's location.

\section{Methods}

For neuromonitoring, a standardized institutional setup was used that included brainstem auditory evoked potentials (BAEPs), SEPs of median and tibial nerves, and MEPs of muscle pairs of upper and lower extremities as well as of corticobulbar muscles. Additionally, free-running electromyography (EMG) of at least the bilateral orbicularis oris and masseter muscles was recorded. In later investigations, bilateral genioglossus muscle EMG was also used. Facial and pharyngeal muscle EMG was added when considered necessary. Direct nerve stimulation (DNS) of CNs with a bipolar concentric probe was used at the surgeons' discretion. All setups followed international recommendations as described previously. ${ }^{17,23,32,33}$ For recording, stimulation, and storage for post hoc analysis, a commercial IOM system (Ewacs System until 2004, since 2003 the ISIS system; both from Inomed Co.) was used.

For intraoperative neuromonitoring, cranial electrode setups followed the international 10-20-electroencephalography system. For stimulation and recording, stainless steel subdermal electroencephalography needle electrodes (various companies) were used.

\section{Setup of SEP}

The SEPs were performed with needle electrode stimulation of the median nerve at the wrist and of the tibial nerve at the medial malleolus. Stimulation was performed using a square pulse with a width of $0.2-0.5 \mathrm{msec}$, an intensity of 20-40 mA, and a frequency of 3.7-5.7 Hz. The recording sites were at $\mathrm{CP} 3, \mathrm{CP} 4, \mathrm{CPz}$, and high cervical (or alternative ipsilateral mastoid electrodes), all referenced to Fz. Responses were recorded with a filter setting of 30-1000 Hz and a sweep length of $160 \mathrm{msec}$.

\section{Setup of MEP}

The MEPs were elicited with transcranial electric stimulation with the train-of-five technique (5 pulses with an individual pulse width of $0.5 \mathrm{msec}$ and an interstimulus interval of $2-4 \mathrm{msec}$ ). The stimulation electrodes were positioned at $\mathrm{C} 4, \mathrm{C} 2, \mathrm{Cz}, \mathrm{C} 1, \mathrm{C} 3$, and $6 \mathrm{~cm}$ anterior to $\mathrm{Cz}$, allowing multiple stimulation electrode montages. Mainly, C1-anode/C2-cathode and C3-anode/Cz-cathode arrangements were used for left hemispheric stimulation and $\mathrm{C} 2$-anode/C1-cathode or $\mathrm{C} 4$-anode/Cz-cathode were used for right hemispheric stimulation; $\mathrm{C} 3 / \mathrm{C} 4$ and $\mathrm{C} 4 / \mathrm{C} 3$ were avoided.

The MEPs were recorded from bilateral abductor pollicis brevis or first interdigitorum muscles and tibial anterior or abductor hallucis muscles. Additional recordings included contralateral extensor digitorum brevis, facial, and pharyngeal muscles, if applicable. For monitoring of extremity muscles, the setup with the lowest motor threshold was chosen and the stimulation intensity was set to $20 \%$ of the highest upper-extremity motor threshold. Responses were recorded with a filter setting of $5-1000 \mathrm{~Hz}$ and a sweep length of $160 \mathrm{msec}$.

\section{Criteria for Warning Signs of Intraoperative Neuromonitoring Modalities \\ Somatosensory Evoked Potentials}

Amplitude decrements of more than 50\% in 3 consecutive recordings were considered significant. Those criteria have been deduced from experimental and clinical studies, which demonstrated that permanent changes exceeding the ones described above are very likely to result in neurological deficits. These parameters have been summarized in international recommendations on intraoperative SEP neuromonitoring. ${ }^{23}$

\section{Motor Evoked Potentials}

In MEPs, an increase of the stimulation intensity required to elicit a response of more than $20 \mathrm{~mA}$ or an amplitude decrement of more than $50 \%$ were considered significant warning signs. The warning criteria for MEPs followed empirical evidence and have recently been refined. 9,17

\section{Brainstem Auditory Evoked Potentials}

Increase in latency of waves III and IV/V for more than $0.5 \mathrm{msec}$ and an amplitude decrement of more than $50 \%$ in BAEPs were considered critical in BAEP monitoring.

\section{Electromyography}

The free-running EMG was observed and spontaneous induced activity was visually rated into A-, B-, and C-trains, according to Romstöck et al. ${ }^{25,26,30}$

\section{Direct Nerve Stimulation of $\mathrm{CNs}$}

Direct cranial nerve stimulation (CN-DNS) was performed using a bipolar concentric probe with a 3-mm diameter and the lowest stimulation possible (0.05-0.5 mA), guided by the surgical aspect of the stimulation site. The compound muscle action potential was categorized into present/absent, as well as by the changes in latencies and 
amplitudes between baseline and stimulation responses during and after tumor resection.

\section{Management of Neurophysiological IOM}

Free-running EMG was evaluated with regard to induced spontaneous activity. The BAEPs, MEPs, and SEPs reflecting the neuronal pathways and their vascular supply in proximity to the resection area were continuously monitored in an alternating mode and only stopped for DNS. All other EPs were intermittently recorded in 5- to 30-minute intervals. Whenever alterations were observed, the modalities serving as controls were repeated to rule out general effects. Warning signs (as described above) alerted surgeons to significant alterations of neuromonitoring.

Surgical response to IOM alterations included reconsiderations of the recent surgical steps. If possible, immediate action was taken; e.g., irrigation with warm saline solution, increase of blood pressure, application of papaverine or nimodipine, intermission of resection, and, when necessary, modification of the surgical strategy.

All EPs were monitored in optimized intervals: EPs were monitored intensively during surgical steps that contained possible neuronal damage, such as direct manipulation of the brainstem and/or vessels possibly providing brainstem supply. On the other hand, EPs were monitored in longer intervals during surgical steps that were unlikely to damage neuronal structures, such as during craniotomy or approach to the lesion.

\section{Anesthesia}

Induction of anesthesia was performed with bolus doses of propofol $(1.5 \mathrm{mg} / \mathrm{kg}) /$ remifentanil $(1 \mu \mathrm{g} / \mathrm{kg})$ and medium-acting muscle relaxant rocuronium $(50-\mathrm{mg}$ bolus). Anesthesia was maintained with propofol (3-6 mg/ $\mathrm{kg} / \mathrm{hr})$ and remifentanil $(0.2-0.3 \mu \mathrm{g} / \mathrm{kg} / \mathrm{min})$.

\section{Clinical Data}

Epidemiological data, including lesion histopathology and location, were prospectively sampled and correlated with postoperative neurological assessment and IOM data.

\section{Clinical Assessment}

Patients' neurological findings were available pre- and postoperatively (immediately postoperatively and at discharge) and assessed based on the patients' charts. Clinical symptoms were described as new $\mathrm{CN}$ or motor/sensory deficits. New deficits were classified as "unexpected" or "truly predicted by IOM changes."

\section{Data Analysis}

Data were extracted from prospective databases maintained by 2 high-volume European tertiary care neurosurgical centers. Between 2002 and 2014, all patients who were monitored during posterior fossa surgery by a single senior neurophysiologist were included. Neurophysiological IOM data, patient demographic data, and data on lesion location and histopathology were centrally collected, reviewed, and completed by chart review when necessary.

Besides descriptive statistics, chi-square and Cramer's
$\mathrm{V}$ test were used to analyze significant differences and correlations. Based on the sample size, the statistical levels of significance stipulated were 0.05 and 0.01 , respectively, for significance and high significance. All analysis was performed with IBM SPSS Statistics software, version 19 (IBM Corp.).

\section{Results}

\section{Demographic Data and General Statistics}

All 305 patients who were identified as eligible were included in the final analysis. The mean patient age was 50.6 \pm 14.1 years, and 166 women and 139 men were included. General data on IOM course and classification of IOM data and neurological outcomes are summarized in Table 1.

\section{Overall Incidence and Distribution of IOM Alterations}

Alterations in IOM were stratified by long-tract (results of MEPs and SEPs) and CN monitoring alterations (results of BAEP, EMG, and CN-DNS) and were grouped by whether or not a corresponding deficit resulted. Additionally, patients with new deficits without IOM alterations were grouped to the modality that failed to detect alterations.

Overall, 173 IOM alterations were observed in 305 cases (56.7\%). Because concomitant alterations of long-tract and $\mathrm{CN}$ monitoring were observed in 15 cases $(4.9 \%)$, the overall incidence of any IOM alteration was 158 of 305 cases $(51.8 \%)$.

In 130 of 305 cases ( $42.6 \%$ of patients; $75.1 \%$ of all IOM alterations), $\mathrm{CN}$ and/or BAEP and CN-DNS alterations were observed, and MEP/SEP alterations were seen in 43 cases (14.0\% of patients; $24.8 \%$ of all IOM alterations). Alterations were followed by neurological sequelae in 98 of 305 cases (36.3\%); this results in $62 \%$ of IOM alterations observed being followed by a neurological deficit.

Sensitivity and specificity for detection of $\mathrm{CN}$ deficits were $98 \%$ and $77 \%$, respectively (positive predictive value [PPV] 71\%, NPV 99\%). Sensitivity and specificity for detection of long-tract deficits were $95 \%$ and $85 \%$, respectively (PPV 48\%, NPV 99\%).

TABLE 1. Classification of IOM courses and observed incidence

\begin{tabular}{|c|c|c|}
\hline IOM \& Clinical Postop Course & Count & $\%$ \\
\hline \multicolumn{3}{|l|}{ IOM unaltered } \\
\hline No new deficit & 128 & 42.0 \\
\hline Unexpected deficits* & 19 & 6.2 \\
\hline \multicolumn{3}{|l|}{ Long-tract MEP/SEP alterations } \\
\hline No new deficit & 24 & 7.9 \\
\hline Long-tract deficit & 19 & 6.2 \\
\hline \multicolumn{3}{|l|}{ AEP, CN MEP, DNS alterations } \\
\hline No new deficit & 36 & 11.8 \\
\hline AEP, $C N$, or single-nerve deficit & 79 & 25.9 \\
\hline Total & 305 & 100.0 \\
\hline $\begin{array}{l}\text { * Unexpected deficits contributed to postope } \\
\text { patients), cerebellar symptoms ( } 3 \text { patients), } \\
\text { ( } 4 \text { patients), dysphasia ( } 1 \text { patient), and delaye } \\
\text { MRI-defined alterations ( } 5 \text { patients). }\end{array}$ & $\begin{array}{l}\text { eficits th } \\
\text { e or uns }\end{array}$ & $\begin{array}{l}\text { hage } 6 \\
\text { nitored } \\
\text { d without }\end{array}$ \\
\hline
\end{tabular}

J Neurosurg Volume 126 • January 2017 


\section{Distribution of IOM Alterations With Regard to Pathology Location}

The posterior fossa allows very limited maneuverability. Neurovascular structures are not only at risk during resection, but also during the approach; e.g., by brain retraction or brain shift. To identify these effects, the exact locations of the lesions included were recorded and clustered to anatomical regions (Table 2 and Fig. 1). Lesion location and histopathology showed a highly significant correlation (Cramer's V $=0.569 ; \mathrm{p}<0.01$ ).

Operations of intraparenchymal lesions or on the surface of the brainstem had the highest probability for any IOM alterations ( $84.6 \%$ of cases), followed by petroclival $(69.2 \%)$ and cerebellopontine $(68.1 \%)$ surgeries. These "big 3" locations and the possible implications of these findings are discussed in more detail in the Discussion section.

Operations in the cerebellopontine angle (CPA) had the highest probability of CN IOM alterations (66.4\%), whereas brainstem surgeries showed the highest probability of MEP/SEP alterations (53.8\%). These 2 locations were also the ones with a high chance of IOM alterations being followed by a corresponding neurological deficit $(81.2 \%$ in the brainstem and $72.2 \%$ in the CPA). Unexpected neurological deficits were most frequently observed during surgery at the cranio-cervical junction, the tentorium, and the petroclival region. The comprehensive data for distribution of IOM alterations per pathology location are provided in Table 2.

\section{Differences in IOM Changes Between Lateral and Midline Lesions}

A significant difference is observed between lateral and midline lesions regarding the likelihood of IOM alterations $(p=0.011)$. In midline pathologies, MEP/SEP altera- tions that did not result in new deficits were observed more often and $\mathrm{CN}$ monitoring alterations followed by deficits were observed less often than stochastically expected.

\section{Distribution of IOM Alterations With Regard to Histopathology}

Biological behavior, mainly defined by histopathology, most likely results in different lesions having a specific risk for IOM alterations and neurological sequelae. Alterations in IOM were correlated with location and histopathology of lesions (both $\mathrm{p}<0.01$; Cramer's $\mathrm{V}=0.327$ ). Alterations in CN IOM were observed most often in schwannomas (76.1\% of surgeries), and $87.0 \%$ of IOM alterations in schwannomas correctly predicted corresponding postoperative $\mathrm{CN}$ deficit.

Changes in MEP/SEP indicating hazard for the long tracts were most frequently observed in cavernomas $(50.0 \%)$, followed by hemangioblastomas (36.4\%). Changes in IOM during cavernoma surgery have a high detection rate for postoperative deficits (85.7\%). These findings are most likely linked to the fact that cavernomas in the posterior fossa are frequently located in the brainstem. New neurological deficits without IOM alterations were most frequently observed in meningioma surgeries $(11.6 \%$ of cases); the overall frequency of this event was $6.2 \%$.

\section{Incidence of Simultaneous IOM Alterations in MEP/SEP and BAEP/CN-DNS}

Alterations in long-tract and $\mathrm{CN}$ monitoring were observed during 15 operations. The highest frequencies were seen in the petroclival $(n=5)$ and brainstem $(n=3)$ locations. Accordingly, meningiomas $(\mathrm{n}=6)$ and cavernomas $(\mathrm{n}=3)$ were the histopathological types in which simultaneous long-tract and $\mathrm{CN}$ alterations were seen most fre-

TABLE 2. Distribution of IOM alterations per pathology location*

\begin{tabular}{|c|c|c|c|c|c|c|c|c|}
\hline Location & $\begin{array}{l}\text { Frequency } \\
\text { (overall \%) }\end{array}$ & $\begin{array}{c}\text { Cases w/ IOM } \\
\text { Alterations ( } \% \\
\text { of surgeries) }\end{array}$ & $\begin{array}{c}\text { BAEP/EMG/ } \\
\text { CN-DNS } \\
\text { Alterations } \\
(\%)\end{array}$ & $\begin{array}{c}\text { BAEP/EMG/ } \\
\text { CN-DNS IOM } \\
\text { Alterations w/ } \\
\text { Corresponding } \\
\text { Deficit (\%) }\end{array}$ & $\begin{array}{c}\text { BAEP/EMG/ } \\
\text { CN-DNS IOM } \\
\text { Alterations w/o } \\
\text { Corresponding } \\
\text { Deficit (\%) }\end{array}$ & $\begin{array}{c}\text { MEP/SEP } \\
\text { Alterations } \\
(\%)\end{array}$ & $\begin{array}{l}\text { MEP/SEP IOM } \\
\text { Alterations w/ } \\
\text { Corresponding } \\
\text { Deficit (\%) }\end{array}$ & $\begin{array}{c}\text { MEP/SEP IOM } \\
\text { Alterations w/o } \\
\text { Corresponding } \\
\text { Deficit (\%) }\end{array}$ \\
\hline $\begin{array}{l}\text { Brainstem (caver- } \\
\text { noma) }\end{array}$ & $13(4.3)$ & $11(84.6)$ & $7(53.8)$ & $6(46.2)$ & $1(7.7)$ & $7(53.8)$ & $6(46.2)$ & $1(7.7)$ \\
\hline Petroclival & $26(8.5)$ & $18(69.2)$ & $14(53.8)$ & $12(46.2)$ & $2(7.7)$ & $9(34.6)$ & $6(23.1)$ & $3(11.5)$ \\
\hline CPA & $116(38.0)$ & 79 (68.1) & $77(66.4)$ & 57 (49.1) & $20(17.2)$ & $4(3.4)$ & $2(1.7)$ & $2(1.7)$ \\
\hline Miscellaneous & $10(3.3)$ & $6(60.0)$ & $6(60.0)$ & $6(60.0)$ & $0(0.0)$ & $2(20.0)$ & $1(10.0)$ & $1(10.0)$ \\
\hline Fourth ventricle & $44(14.4)$ & $23(52.3)$ & $14(31.8)$ & $6(13.6)$ & $8(18.2)$ & $11(25.0)$ & $4(9.1)$ & $7(15.9)$ \\
\hline $\begin{array}{l}\text { Cranio-cervical } \\
\text { junction }\end{array}$ & $2(0.7)$ & $1(50.0)$ & $1(50.0)$ & $0(0.0)$ & $1(50.0)$ & $0(0.0)$ & $0(0.0)$ & $0(0.0)$ \\
\hline Brainstem (other) & $11(3.6)$ & $5(45.5)$ & $3(27.3)$ & $1(9.1)$ & $2(18.2)$ & $3(27.3)$ & $0(0.0)$ & $3(27.3)$ \\
\hline Petrous & $7(2.3)$ & $3(42.9)$ & $3(42.9)$ & $1(14.3)$ & $2(28.6)$ & $0(0.0)$ & $0(0.0)$ & $0(0.0)$ \\
\hline Tentorium & $23(7.5)$ & $6(26.1)$ & $3(13.0)$ & $3(13.0)$ & $0(0.0)$ & $3(13.0)$ & $0(0.0)$ & $3(13.0)$ \\
\hline Cerebellar & $52(17.0)$ & $6(11.5)$ & $2(3.8)$ & $0(0.0)$ & $2(3.8)$ & $4(7.7)$ & $0(0.0)$ & $4(7.7)$ \\
\hline Vascular & $1(0.3)$ & $0(0.0)$ & $0(0.0)$ & $0(0.0)$ & $0(0.0)$ & $0(0.0)$ & $0(0.0)$ & $0(0.0)$ \\
\hline Overall & $305(100.0)$ & $158(51.8)$ & $130(42.6)$ & $92(30.1)$ & 38 (12.5) & $43(14.1)$ & $19(6.2)$ & $24(7.9)$ \\
\hline
\end{tabular}

* In addition to overall and specific frequencies, true positives and false negatives are given for each modality with respect to the specific localization. 


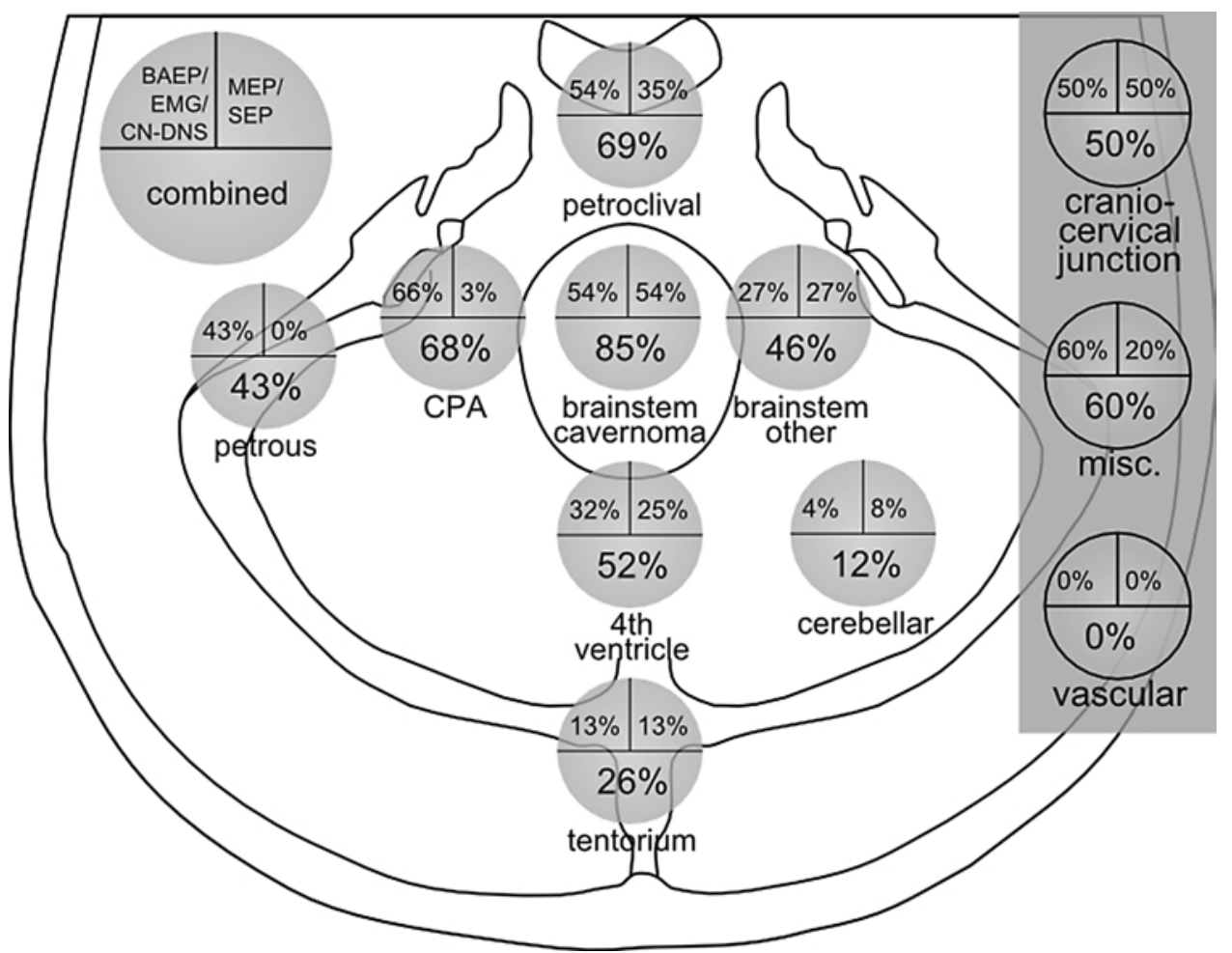

FIG. 1. Schematic of the distribution of BAEP/EMG/CN-DNS and MEP/SEP alterations with respect to pathology location. Note that concomitant alterations occur in certain locations, resulting in the combined percentage being smaller than the sum of BAEP/ EMG/CN-DNS and MEP/SEP. Locations with no events have been omitted.

quently. Combined IOM alterations commonly resulted in combined long-tract and $\mathrm{CN}$ deficits $(66 \%)$ and therefore most likely represent serious incidents during surgery (e.g., critical changes to blood supply or direct nervous tissue damage).

\section{Additional Analysis for Possible Confounding Factors}

Additional analysis was performed for sex, contributing center, and age, with age being dichotomized at 65 years. The mean age was 50.6 years; 242 patients were older than 65 years. One hundred thirty-nine patients were men and 166 were women; 208 patients were from the first center and 97 patients were from the second center. No significant differences were observed in any of these variables regarding distribution of IOM alterations.

\section{Discussion}

To the best of our knowledge, this is currently the largest series analyzing more than 300 patients undergoing posterior fossa surgery with a standardized setup of neurophysiological IOM containing BAEPs, CN-DNS, and free-running EMG of at least 2 bilateral CNs, SEPs, and MEPs.

This extensive setup, which was used regardless of the lesion location and the empirically expected likelihood of alterations, allows the analysis of the probability of IOM alterations with respect to lesion location and histopathological type. We demonstrated that alterations of SEPs and MEPs are commonly observed during surgery in the posterior fossa. Long-tract monitoring is therefore recommended as a valuable addition to $\mathrm{CN}$ monitoring during these surgeries. The comprehensive data allow an estimation of the likelihood of IOM alterations based on the lesion's location-information that is crucial for surgical planning and tailored IOM setups.

\section{Distribution of IOM Alterations With Regard to Lesion Location and Histopathology}

The importance of MEP/SEP monitoring and its clinical implications during posterior fossa surgery has not been investigated intensively before. Intraoperative monitoring requires limited technical, human, and financial resources; the use of IOM should therefore be justified by scientific data. This analysis was designed to serve as a guide for surgeons and neurophysiologists to set up IOM depending on the lesion's location. We did this by providing information on which IOM modality is necessary for which lesion, and which procedures might be performed without or with reduced IOM assistance. Additionally, possible interpretations of IOM alterations grouped for MEP/SEP and CN data are provided for each localization type. These data are crucial when confronted with IOM alterations during surgery.

Tumor location and tumor histopathology within the posterior fossa are closely linked. The histopathological type is often unknown at the beginning of and during surgery. Our data demonstrate that tumor location influences 
the likelihood of long-tract IOM alteration, and MEP/SEP monitoring should therefore be performed based on tumor location. In particular, removal of tumors located at the petroclival face of the posterior fossa or at the tentorium was associated not only with a higher incidence of $\mathrm{CN}$ deficits, but also with alterations in MEPs/SEPs. The latter are likely to be followed by devastating impairments.

\section{The "Top 3" Locations}

In our analysis, the brainstem, the CPA, and the petroclival region were the top 3 locations regarding overall IOM alterations. Brainstem lesions, especially brainstem cavernomas, had a high incidence of $\mathrm{CN}$ and long-tract IOM alterations, both of which were frequently followed by neurological deficits. These observations are easily explained by the high density of important tracts and nuclei in this region and their challenging blood supply. A complete IOM setup is commonly used during these surgeries, an approach once more confirmed by our findings, in that IOM warning signs allow changes in the surgical strategy (e.g., halt of resection, rinsing with saline with or without nimodipine, or increase of blood pressure).

Meningiomas and chordomas are mainly seen in the petroclival region. This region also presented with a high incidence of $\mathrm{CN}$ and long-tract IOM alterations, although these alterations were less likely to be followed by a corresponding deficit. The IOM alterations observed are more likely to have been induced by nerve or tissue traction rather than by direct tissue damage, as seen in brainstem lesions. Changes in blood flow (especially venous outflow) might additionally contribute to this observation. Changes in surgical strategy (as mentioned above, plus reduction of traction/pressure) as a consequence of IOM alterations might play a more significant role in this location when compared with brainstem surgery.

As one might expect, high numbers of IOM alterations are seen during CPA surgery. These changes are almost exclusively seen in $\mathrm{CN}$ monitoring, but long-tract changes were observed in $3.4 \%$ of cases and were followed by a corresponding deficit in $1.7 \%$ of cases. Although these numbers are low, we do include MEP/SEP monitoring during these surgeries, because MEP/SEP monitoring provides information on the depth of anesthesia and therefore facilitates interpretations of $\mathrm{CN}$ (especially BAEP) monitoring changes.

\section{Changes in IOM in Lateral Versus Midline Lesions}

These findings are related to surgical manipulation during the approach and the likelihood of midline lesions to be intraaxial (e.g., in the brainstem). Drainage of CSF and mild brain retraction applied to the cerebellum and $\mathrm{CNs}$ are required to access the brain midline structures, frequently resulting in $\mathrm{CN}$ IOM alteration. We found that these alterations resulted in a lower than expected probability of $\mathrm{CN}$ deficits. This might be explained by increased awareness, because even slight CN IOM alterations were immediately followed by changes in the surgical strategy.

A second possible explanation for the increased frequency of IOM alteration and neurological sequelae observed in midline pathologies is that dissection of arachnoidal adhesions includes coagulation of small vessels, not all of which are clearly without function for the brain. Meningiomas in the posterior fossa are commonly located in proximity to the deep venous systems, and the deficits observed were mostly linked with postoperative unexpected edema or hemorrhage. In cases resulting in changes to deep venous drainage, the predictive value of IOM may be limited because functional alterations might be delayed. These limitations could possibly be overcome by extension of the neuromonitoring period long into the postoperative period. In the case of meningiomas, arachnoid membranes are commonly thinned out, and tighter adhesion between the tumor and the brain or brainstem usually exists. ${ }^{5,6,19}$ Therefore, surgeries in which tumor dissection from the brain and brainstem is necessary carry a higher risk than surgeries in which less dissection is required. In extensive approaches, coagulation might add up to decreased venous and/or arterial flow and result in temporary or permanent ischemia and postoperative edema.

\section{Concomitant BAEP/EMG/CN-DNS and MEP/SEP Changes}

Concomitant changes in $\mathrm{CN}$ and long-tract monitoring were most often observed during surgery in the petroclival region and the brainstem, and were followed by neurological sequelae in a high percentage of cases. This can be caused by damage to the brainstem's blood supply or by direct long-tract and $\mathrm{CN}$ damage. Additionally, adhesions to the brainstem and CNs are commonly found in petroclival lesions.

\section{Overall Incidence and Distribution of IOM Alterations}

The relationship between changes in neurophysiological IOM modalities and clinical outcome has been extensively described. ${ }^{7,8,20,28}$ We therefore simplified our analysis, not taking the duration and detailed cause (and course) of IOM alteration into consideration. It was anticipated that alterations not resulting in neurological sequelae were either transient (i.e., reversed by action taken) or real false positives. Therefore, sensitivity, specificity, PPV, and NPV are skewed toward NPV and specificity. From the surgeon's and neurophysiologist's perspective, the overall incidence of intraoperative alterations is important because, at the time of their occurrence, their duration is not known. Each alteration can be permanent. Therefore, any alteration potentially resulting in a neurological deficit should be addressed by the surgeon and/or the anesthesia team. An experienced team is necessary to allow adequate interpretation and timely response to any IOM alteration observed. One should keep in mind that MEP/SEP monitoring delivers information beyond the pure long-tract monitoring; e.g., depth of anesthesia and rare incidents outside of the surgical field such as stroke, remote bleeding, or nerve damage caused by a patient's position.

Overall, MEP/SEP alterations were observed less frequently than CN/BAEP/CN-DNS alterations, and were less likely to result in neurological deficits: 19 of $43 \mathrm{MEP} /$ SEP alterations (44\%) but 92 of $130 \mathrm{CN} / \mathrm{BAEP} / \mathrm{CN}-\mathrm{DNS}$ alterations (71\%) were followed by early neurological deficits. The overall higher rate of alterations in CN/BAEP/ CN-DNS might be explained by the fact that CNs are very sensitive to any kind of manipulation. Direct or indirect manipulation (mechanical forces such as stretching or 
compression, irrigation, use of bipolar electrocauterization, tumor removal with a Cavitron ultrasonic surgical aspirator) is very likely to result in a nerve conduction block and/or any kind of spontaneous activity in EMG. The duration of nerve conduction block and its resolution are very difficult to estimate at the time of the occurrence.

For AEPs, warning criteria are well established and allow for timely warning..$^{13-16,29}$ On the other hand, due to the underlying disease, BAEP changes might be unavoidable. Therefore, the surgical aim of complete tumor resection in vestibular schwannoma creates a bias toward a higher incidence of BAEP alterations. One has to keep in mind that these are included in $\mathrm{CN}$ alterations in our analysis.

\section{Additional Analysis for Possible Confounding Factors}

Interestingly, we could not demonstrate any confounding factors. The influence of positioning has been described in previous studies. ${ }^{34}$ Because Institution 1 predominantly used the semisitting position and Institution 2 the modified park bench position, we expected that this would affect IOM alterations without corresponding deficits. This was not the case. Most likely, analysis was biased by the empirical knowledge of IOM changes thought to be related to the semisitting position. Therefore, certain IOM alterations were attributed to the position and not reported as relevant.

\section{Conclusions}

The incidence of IOM alterations during surgery in the posterior fossa varies widely among different lesions, locations, and histopathological types. This analysis provides crucial information on the necessity of IOM, especially MEP/SEP monitoring, during posterior fossa surgery. Because MEP/SEP and CN IOM alterations were common during posterior fossa surgery in this large series, we recommend the simultaneous use of both modalities.

\section{Acknowledgments}

This study was financed by the Department of Neurosurgery, University Hospital of the Johann Wolfgang Goethe University, Frankfurt, Germany.

\section{References}

1. Dong CC, Macdonald DB, Akagami R, Westerberg B, Alkhani A, Kanaan I, et al: Intraoperative facial motor evoked potential monitoring with transcranial electrical stimulation during skull base surgery. Clin Neurophysiol 116:588-596, 2005

2. Engler GL, Spielholz NJ, Bernhard WN, Danziger F, Merkin $\mathrm{H}$, Wolff T: Somatosensory evoked potentials during Harrington instrumentation for scoliosis. J Bone Joint Surg Am 60:528-532, 1978

3. Gläsker S, Pechstein U, Vougioukas VI, Van Velthoven V: Monitoring motor function during resection of tumours in the lower brain stem and fourth ventricle. Childs Nerv Syst 22:1288-1295, 2006

4. Guo L, Gelb AW: The use of motor evoked potential monitoring during cerebral aneurysm surgery to predict pure motor deficits due to subcortical ischemia. Clin Neurophysiol 122:648-655, 2011

5. Hafez A, Nader R, Al-Mefty O: Preservation of the superior petrosal sinus during the petrosal approach. J Neurosurg 114:1294-1298, 2011

6. Kaku S, Miyahara K, Fujitsu K, Hataoka S, Tanino S, Okada $\mathrm{T}$, et al: Drainage pathway of the superior petrosal vein evaluated by CT venography in petroclival meningioma surgery. $\mathbf{J}$ Neurol Surg B Skull Base 73:316-320, 2012

7. Kang DZ, Wu ZY, Lan Q, Yu LH, Lin ZY, Wang CY, et al: Combined monitoring of evoked potentials during microsurgery for lesions adjacent to the brainstem and intracranial aneurysms. Chin Med J (Engl) 120:1567-1573, 2007

8. Kodama K, Javadi M, Seifert V, Szelényi A: Conjunct SEP and MEP monitoring in resection of infratentorial lesions: lessons learned in a cohort of 210 patients. J Neurosurg 121:1453-1461, 2014

9. Kombos T, Suess O, Ciklatekerlio O, Brock M: Monitoring of intraoperative motor evoked potentials to increase the safety of surgery in and around the motor cortex. J Neurosurg 95:608-614, 2001

10. Kombos T, Suess O, Funk T, Kern BC, Brock M: Intraoperative mapping of the motor cortex during surgery in and around the motor cortex. Acta Neurochir (Wien) 142:263268, 2000

11. Kothbauer KF: Intraoperative neurophysiologic monitoring for intramedullary spinal-cord tumor surgery. Neurophysiol Clin 37:407-414, 2007

12. Kothbauer K, Deletis V, Epstein FJ: Intraoperative spinal cord monitoring for intramedullary surgery: an essential adjunct. Pediatr Neurosurg 26:247-254, 1997

13. Legatt AD: Mechanisms of intraoperative brainstem auditory evoked potential changes. J Clin Neurophysiol 19:396-408, 2002

14. Legatt AD, Emerson RG: Motor evoked potential monitoring-it's about time. J Clin Neurophysiol 19:383-386, 2002

15. Levine RA, Ojemann RG, Montgomery WW, McGaffigan PM: Monitoring auditory evoked potentials during acoustic neuroma surgery. Insights into the mechanism of the hearing loss. Ann Otol Rhinol Laryngol 93:116-123, 1984

16. Loftus CM, Traynelis VC: Intraoperative Monitoring Techniques in Neurosurgery. New York: McGraw-Hill, 1994

17. MacDonald DB: Intraoperative motor evoked potential monitoring: overview and update. J Clin Monit Comput 20:347-377, 2006

18. MacDonald DB, Al Zayed Z, Khoudeir I, Stigsby B: Monitoring scoliosis surgery with combined multiple pulse transcranial electric motor and cortical somatosensory-evoked potentials from the lower and upper extremities. Spine (Phila Pa 1976) 28:194-203, 2003

19. Morisako H, Goto T, Chokyu I, Ishibashi K, Ohata K: Preoperative evaluation of the petrosal vein with contrast-enhanced PRESTO imaging in petroclival meningiomas to establish surgical strategy. Neurol Med Chir (Tokyo) 53:490-495, 2013

20. Neuloh G, Bogucki J, Schramm J: Intraoperative preservation of corticospinal function in the brainstem. J Neurol Neurosurg Psychiatry 80:417-422, 2009

21. Neuloh G, Pechstein U, Schramm J: Motor tract monitoring during insular glioma surgery. J Neurosurg 106:582-592, 2007

22. Neuloh G, Schramm J: Monitoring of motor evoked potentials compared with somatosensory evoked potentials and microvascular Doppler ultrasonography in cerebral aneurysm surgery. J Neurosurg 100:389-399, 2004

23. Nuwer MR, Daube J, Fischer C, Schramm J, Yingling CD: Neuromonitoring during surgery. Report of an IFCN Committee. Electroencephalogr Clin Neurophysiol 87:263-276, 1993

24. Nuwer MR, Dawson EG, Carlson LG, Kanim LE, Sherman JE: Somatosensory evoked potential spinal cord monitoring reduces neurologic deficits after scoliosis surgery: results of 
a large multicenter survey. Electroencephalogr Clin Neurophysiol 96:6-11, 1995

25. Romstöck J, Strauss C, Fahlbusch R: Continuous electromyography monitoring of motor cranial nerves during cerebellopontine angle surgery. J Neurosurg 93:586-593, 2000

26. Romstöck J, Strauss C, Fahlbusch R: Identification of cranial nerve nuclei. Muscle Nerve 23:1445-1446, 2000

27. Sala F, Lanteri P, Bricolo A: Motor evoked potential monitoring for spinal cord and brain stem surgery. Adv Tech Stand Neurosurg 29:133-169, 2004

28. Sarnthein J, Bozinov O, Melone AG, Bertalanffy H: Motorevoked potentials (MEP) during brainstem surgery to preserve corticospinal function. Acta Neurochir (Wien) 153:1753-1759, 2011

29. Sekiya T, Iwabuchi T, Kamata S, Ishida T: Deterioration of auditory evoked potentials during cerebellopontine angle manipulations. An interpretation based on an experimental model in dogs. J Neurosurg 63:598-607, 1985

30. Strauss C, Romstöck J, Fahlbusch R, Rampp S, Scheller C: Preservation of facial nerve function after postoperative vasoactive treatment in vestibular schwannoma surgery. Neurosurgery 59:577-584, 2006

31. Szelényi A, Hattingen E, Weidauer S, Seifert V, Ziemann $\mathrm{U}$ : Intraoperative motor evoked potential alteration in intracranial tumor surgery and its relation to signal alteration in postoperative magnetic resonance imaging. Neurosurgery 67:302-313, 2010

32. Szelényi A, Kothbauer K, de Camargo AB, Langer D, Flamm ES, Deletis V: Motor evoked potential monitoring during cerebral aneurysm surgery: technical aspects and comparison of transcranial and direct cortical stimulation. Neurosurgery 57 (4 Suppl):331-338, 2005
33. Szelényi A, Kothbauer KF, Deletis V: Transcranial electric stimulation for intraoperative motor evoked potential monitoring: Stimulation parameters and electrode montages. Clin Neurophysiol 118:1586-1595, 2007

34. Wiedemayer H, Fauser B, Sandalcioglu IE, Schäfer H, Stolke $\mathrm{D}$ : The impact of neurophysiological intraoperative monitoring on surgical decisions: a critical analysis of 423 cases. J Neurosurg 96:255-262, 2002

\section{Disclosures}

The authors report no conflict of interest concerning the materials or methods used in this study or the findings specified in this paper.

\section{Author Contributions}

Conception and design: Slotty, Javadi, Seifert, Szelényi. Acquisition of data: Abdulazim, Kodama, Javadi, Szelényi. Analysis and interpretation of data: Slotty, Kodama, Hänggi, Szelényi. Drafting the article: Slotty, Szelényi. Critically revising the article: Slotty, Kodama, Hänggi, Seifert, Szelényi. Reviewed submitted version of manuscript: Slotty, Abdulazim, Kodama, Hänggi, Seifert, Szelényi. Approved the final version of the manuscript on behalf of all authors: Slotty. Statistical analysis: Slotty. Administrative/ technical/material support: Javadi, Seifert, Szelényi. Study supervision: Szelényi.

\section{Correspondence}

Philipp J. Slotty, Neurosurgical Department, Heinrich-Heine University Düsseldorf, Moorenstrasse 5, 40225 Düsseldorf, Germany.email: slotty@hhu.de. 